\author{
UCRL-JC-121635 \\ PREPRINT \\ CoNF-9511106-.2
}

\title{
Application of Optical Interconnect Technology at \\ Lawrence Livermore National Laboratory
}

\author{
R. E. Haigh \\ M. E. Lowry \\ K. McCammon \\ R. Hills \\ R. Mitchell \\ D. Sweider
}

\section{RECEIVED \\ OCT 061995

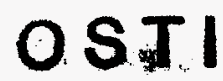

This paper was prepared for submittal to the

IEEE Military Communication Conference

San Diego, CA

November 5-8, 1995

August 10, 1995

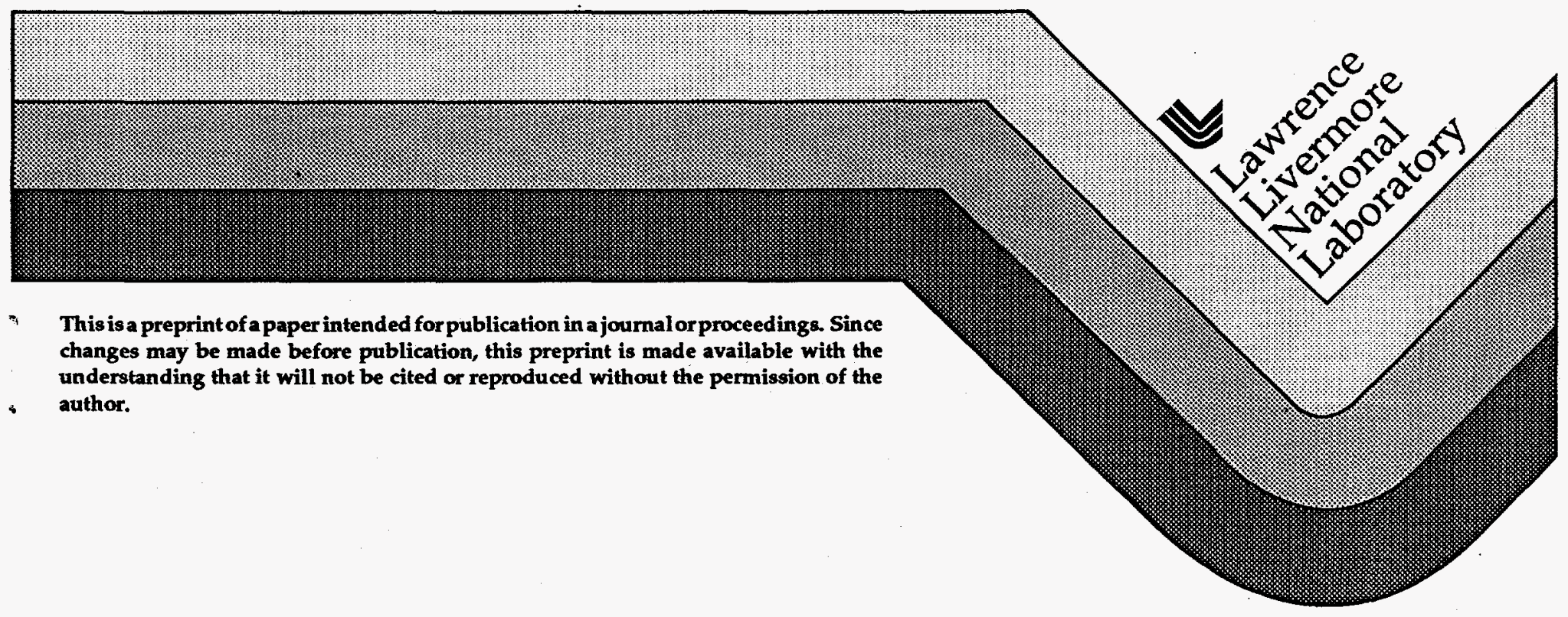

This is a preprint of a paper intended for publication in a journalor proceedings. Since changes may be made before publication, this preprint is made available with the understanding that it will not be cited or reproduced without the permission of the author. 
(t)

This document was prepared as an account of work sponsored by an agency of the United States Government. Neither the United States Government nor the University of California nor any of their employees, makes any warranty, express or implied, or assumes any legal liability or responsibility for the accuracy, completeness, or usefulness of any information, apparatus, product, or process disclosed, or represents that its use would not infringe privately owned rights. Reference herein to any specific commercial product, process, or service by trade name, trademark, manufacturer, or other wise, does not necessarily constitute or imply its endorsement, recommendation, or favoring by the United States Government or the University of California. The views and opinions of authors expressed herein do not necessarily state or reflect those of the United States Government or the University of California, and shall not be used for advertising or product endorsement purposes. 


\section{DISCLAIMER}

Portions of this document may be illegible in electronic image products. Images are produced from the best available original document. 


\title{
Application of Optical Interconnect Technology at Lawrence Livermore National Laboratory
}

\author{
R. E. Haigh, M. E. Lowry, K. McCammon, R. Hills, \\ R. Mitchell, and D. Sweider \\ Lawrence Livermore National Laboratory \\ 7000 East Avenue, L-54, Livermore, California 94551-0808
}

\begin{abstract}
Optical interconnects will be required to meet the information bandwidth requirements of future communication and computing applications. At Lawrrence Livermore National Laboratory, we are involved in applying optical interconnect technologies in two distinct application areas: Multi-Gigabit/sec Computer Backplanes and Gigabit/sec Wide Area Networking using Wavelength Division Multiplexing. In this paper, we discuss our efforts to integrate optical interconnect technologies into prototype computing and communication systems.
\end{abstract}

\section{Parallel Optical Computer Interconnects}

LLNL has teamed with the OETC (Optoelectronic Technology Consortium: AT\&T, Honeywell, IBM, and Lockheed-Martin) on a technology demonstration of parallel optical interconnects. The demonstration involves a prototype of an optically interconnected workstation cluster, under development at LLNL, to evaluate parallel optical interconnects in a high performance computing environment. Our prototyping goals include demonstrating the application, performance, and reliability of optical interconnects in a parallel processing environment comprised of a cluster of commercially available workstations executing real scientific applications.

The workstations in our test bed will be interconnected in a ring topology using the SCI (Scalable Coherent Interface) communications protocol. We are developing a PCI to SCI interface to a workstation as a method of implementing the SCI protocol. A custom $\mathrm{GaAs}$ ASIC, offered commercially by Vitesse Semiconductor, implements the SCI protocol.
The current SCI implementation uses 16 parallel data lines (data rates of $500 \mathrm{Mbits} / \mathrm{s}$ per data line), plus one clock and a strobe line.

The transport layer of the SCI protocol will be implemented using the 32-channel OETC parallel optical interconnect technology ${ }^{0}$. Each channel within the optical interconnect has a communication capacity of $500 \mathrm{Mbits} / \mathrm{second}$, and a single link has a communication bandwidth of $16 \mathrm{Gbits} /$ second, only 18 of the 32 data channels will be required for our prototype SCI application. The SCI workstation cluster, linked with optical interconnects, will extend the usable separation between processing nodes to over 100 meters, initially, enabling distributed cluster computing with workstations across small campuses. To characterize the performance of the workstation cluster, we intend to benchmark variations of parallel processing applications developed at LLNL on the prototype system.

Currently we plan to incorporate 10 workstations into our test bed, each with its own communication link to an adjacent node in the ring as illustrated in figure 1 . Since the communication paths are point-to-point, multiple communications may occur concurrently in the ring allowing for a peak network bandwidth of over $160 \mathrm{Gigabits} / \mathrm{second}$. Once switches are developed to support the SCI communications protocol, SCI networks with substantially higher bisectional bandwidths will be realizable. 


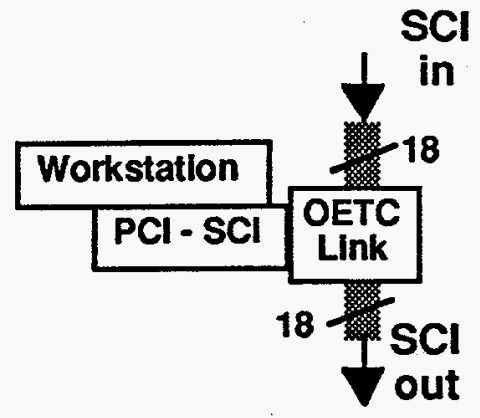

Figure 1. An Optically Interconnected SCI Node in a Workstation Cluster

A number of high performance computer houses are developing implementations of SCI for their next generation products ${ }^{1}$. Additionally, the DoD JAST (Joint Advanced Striker Technology) program has identified the SCI protocol as an enabling technology to achieve a unified avionics architecture for the next generation striker aircraft. Parallel optical interconnects will become a suitable transport layer strategy for next generation SCI and other high performance communication protocols as the interconnect technology becomes commercially available.

\section{Optical Interconnects in Multi-}

Wavelength Transparent Optical

Networks

Next generation, wide area optical networks will employ wavelength division multiplexing along with optical routing and optical amplification to achieve a higher capacity, more affordable, telecommunications information infrastructure. As a member of the NTONC, LLNL is involved in the integration and deployment of experimental optical technologies in the NTONC prototype network which encompasses the San Francisco Bay as illustrated in figure 2. The National Transparent Optical Network Consortium (NTONC) ${ }^{2}$ is an ARPA sponsored consortium led by NORTEL which is developing a four wavelength transparent optical network in the $1550 \mathrm{~nm}$ window. The optical network will also serve as an open test bed to evaluate emerging optoelectronic technologies. The network backbone will have network access nodes initially at Pacbell, Sprint, UC Berkeley, and LLNL. Tributaries to additional user sites will be added to allow real applications to be developed and tested across the network. The NTONC members are Case Western Reserve University, Columbia University, Hughes, LLNL, NORTEL/BNR, Rockwell, Pacific Bell, Sprint, Uniphase Telecommunications Products and United Technologies Research Center.

The optical network is being deployed on the embedded fiber infrastructure of Pacific Bell and Sprint. The fiber is standard non-dispersion shifted fiber; and therefore, dispersion management of bit-rate distance products will be required for directly modulated OC-48 transmitters as the network diameter which exceeds $200 \mathrm{~km}$ is beyond the reach of most directly modulated technologies. Optical amplifiers will be deployed within the Sprint and Pacbell infrastructure to compensate for optical fiber and switching losses in the network.

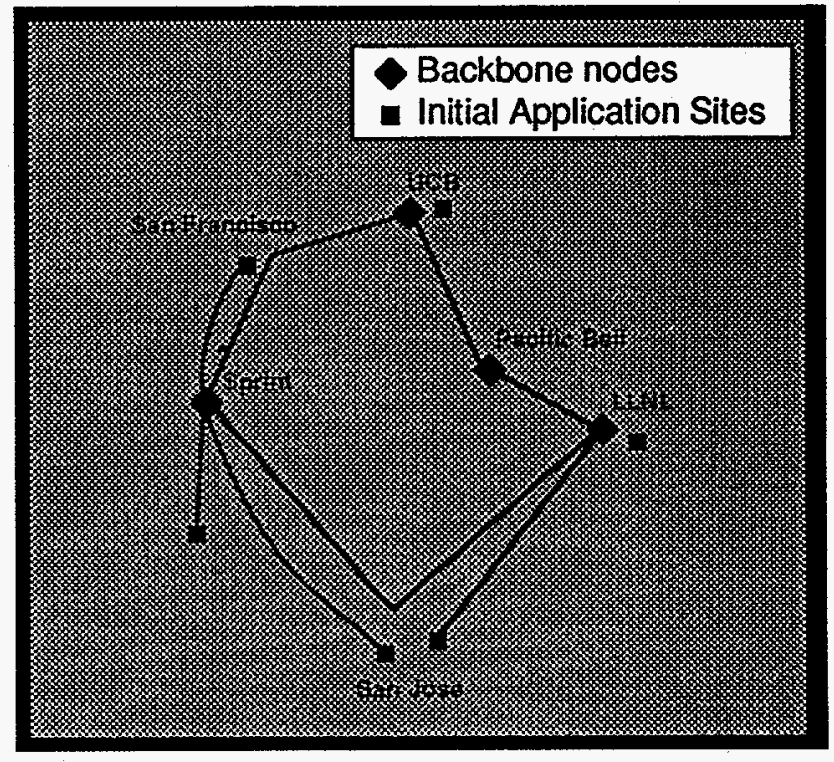

\section{Figure 2. NTONC Transparent Optical Network Field Trial}

One of the requirements necessary to achieve a scalable, multi-wavelength, all optical network involves the ability to switch optical signals, on a wavelength selective basis, and to re-use wavelengths within the network. Wavelength reuse is critical to minimize the number of wavelengths in the network and the associated 
hardware complexity associated with large numbers of wavelengths. Wavelength selective switching of the WDM optical signals will be achieved using a combination of AOTF (acoustooptic tunable filter) and space-division optomechanical technologies. The AOTF technology is being developed by United Technologies Research Center and Uniphase Telecommunications Products and will be an enhanced version of the AOTF technology developed under the ONTC program.

While optical networking technologies offer the promise of delivering on the bandwidth, flexibility, and scalability requirements of future telecommunication environments, economic factors will play a major role in determining the rate at which optical technologies are actually deployed in commercial systems. Members of the NTONC, lead by BNR, are exploring the economic benefits of all optical networking approaches versus traditional telecommunication implementations ${ }^{3}$. The economic tradeoffs include the costs of the optoelectronic technologies, cost of optical vs. electrical switching, and costs incurred in administration and management of all optical vs. traditional communication networks.

The optical network that the NTONC is deploying will provide ATM connectivity between sites using multi-wavelength $\mathrm{OC}-48$ SONET data transport in the backbone supported by ATM switches at the node sites. We will also deploy Subcarrier Multiplexing (SCM) Technology developed by Hughes to demonstrate clear channel functionality. The SCM technology will carry analog video signals between sites in the network. The clear channel capability of the network allows format and protocol independent data to be transported between end users. The clear channel function could be useful for legacy telecommunication protocols as well as providing for an infrastructure to support emerging wireless datacom applications. Finally, many DoD communication requirements may require support for non-standard communication protocols, e.g. satellite to earth communications. We intend to further investigate the technical requirements for networks involving both satellite and optical fiber network connectivity.

\section{Acknowledgments}

We would like to thank W. J. Lennon, L. Thombley, and G. Armstrong at LLNL, Members of the Optoelectonic Technology Consortium and Members of the National Transparent Optical Network Consortium for their helpful discussions and technical support. This work is sponsored by ARPA under the NCAICM and BIT programs. Work performed under the auspices of the U. S. Department of Energy by Lawrence Livermore National Laboratory under Contract W-7405-Eng-48.

\section{References}

'Wong,Y.M., Muehlner,D.J., Faudskar,C.C., Buchholz, D.B., Fusgteyn,M., Brandner, J.L., Parzygnat, W.J., Morgan, R.A., Mullally, T. Leibenguth, R.E., Guth, G.D., Focht, M.W., Glogovsky, K.G., Zilko, J.L., Gates, J.V., Anthony, P.J., Tyrone,Jr., B.H., Ireland, T.J., Lewis,Jr., D.H., Smith, D.F., Nati, S.F., Lewis, D.K., Rogers, D.L., Aispain, H.A., Gowda, S.M., Walker, S.G., Kwark Y.H., Bates, R.J.S., Kuchta, D.M., and Crow, J.D. "Technology Development of a HighDensity 32-channel $16 \mathrm{~Gb} / \mathrm{s}$ Optical Data Link for Optical Interconnection Applications for the Optoelectronic Technology Consortium (OETC)," IEEE Journal of Lightwave Technology, pp.995-1016, June 1995, v13, No 6.

${ }^{1}$ Convex Exemplar paper ( NASA Ames authors) in last SIZZLE-2 proceedings ( March or April '95) see Rob Hills for proceedings / reference info.

${ }^{2}$ Lennon, W.J. and Thombley, R.L., "Two Wavelength Division Multiplexing WAN Trials", Compcon ' 95.

${ }^{3}$ Coathup, L., Lu, C.-Y., and Roorda, P., "Economical Applications of All Optical Networks," IEEE LEOS Summer Topicals 1995, TuB1. 